\title{
EL DOCENTE EN LA EDUCACIÓN INTERCULTURAL BILINGÜE DE LA COMUNIDAD WAORANI: CASO ESCUELA IKA
}

\section{TEACHERS IN THE INTERCULTURAL BILINGUAL EDUCATION OF THE WAORANI COMMUNITY: IKA SCHOOL CASE STUDY}

LORENA ÁLVAREZ E¹., MARÍA ARROYO², LUIS CÁRDENAS P33.

Recibido: 30 de mayo de 2018 Aceptado: 22 de octubre de 2018

\footnotetext{
${ }_{1}^{1}$ Pontificia Universidad Católica del Ecuador, Facultad de Ciencias de la Educación, Quito, Ecuador (malvarez124@puce.edu.ec)

${ }^{2}$ Pontificia Universidad Católica del Ecuador, Facultad de Ciencias de la Educación, Quito, Ecuador (marroyo621@puce.edu.ec).

${ }^{3}$ Pontificia Universidad Católica del Ecuador, Facultad de Ciencias de la Educación, Quito, Ecuador (Icardenas690@puce.edu.ec).
} 
네lㅔ 174

174 


\section{EL DOCENTE EN LA EDUCACIÓN INTERCULTURAL BILINGÜE DE LA COMUNIDAD WAORANI: CASO ESCUELA IKA}

\section{TEACHERS IN THE INTERCULTURAL BILINGUAL EDUCATION OF THE WAORANI COMMUNITY: IKA SCHOOL CASE STUDY}

Lorena Álvarez E., María Arroyo, Luis Cárdenas P.

Palabras clave: Educación Intercultural, Educación Bilingüe, Waorani, Perfil Docente Keywords: Intercultural Education, Bilingual Education, Waorani, Teaching Profile

\section{RESUMEN}

Este proyecto de investigación tiene la finalidad de presentar al lector el contexto educativo de la comunidad waorani, zona alejada de las grandes ciudades e inmersa en la selva amazónica ecuatoriana. Este sector posee cultura e idioma distintos al de las grandes urbes y requiere de docentes con características específicas para desempeñarse en centros de educación intercultural bilingüe.
Con el propósito de identificar las necesidades educativas de los estudiantes en los subniveles de Básica Elemental y Básica Media, de la Escuela Ika, se partió del análisis del entorno escolar de la comunidad Waorani de Guiyero para la construcción de un perfil docente intercultural bilingüe a través de un diseño de investigación teórico-conceptual. 
El perfil del maestro intercultural bilingüe, producto de este trabajo, fue diseñado a través de los cuatro componentes propuestos por el Ministerio de
Educación del Ecuador e incluyó una serie de conductas que se esperan observar en los profesores de la Escuela Ika durante el desempeño de la labor docente.

\section{ABSTRACT}

This research project aims to present the reader with the educational context of the Waorani communityan area distant from the big cities and immersed in the Ecuadorian Amazon rainforest. This sector has a culture and language distinct from metropolitan areas and requires teachers with specific characteristics to perform in bilingual intercultural education centers.

In order to identify the educational needs of the students in the Basic and Elementary Basic sub-levels of the Ika School, the analysis of the school en- vironment of the Waorani community of Guiyero was used as basis for the construction of a bilingual intercultural teaching profile through a theoretical-conceptual research design.

The profile of the intercultural bilingual teacher produced by this work was designed through the four components proposed by the Ministry of Education of Ecuadorm It included a series of behaviors that should be observed in the teachers of the Ika School when carrying out their teaching duties.

\section{INTRODUCCIÓN}

En el Parque Nacional Yasuní (PNY), área natural del Ecuador ubicada en las provincias de Orellana y Pastaza, se localizan algunas comunidades de la nacionalidad Waorani. En este sector se encuentran las poblaciones de Guiyero, Ganketapare, Paraíso, Timpoka y Peneno, cuyos niños acuden al Centro Educativo Intercultural Bilingüe CECIB-Ika. Esta institución educativa de carácter pluridocente funciona en jornada matutina y ofrece educación formal en los sub niveles de Educación General Básica (EGB) preparatoria, elemental y media.

En la Escuela Ika los maestros imparten clases únicamente en lengua castellana, abordan contenidos ajenos a la cultura waorani propuestos por los textos 
escolares que reciben del Ministerio de Educación y manejan estrategias didácticas enfocadas en la mecanización de procesos y la memorización de contenidos (PUCE, 2016). Lo citado trae como consecuencia que los estudiantes reciban una deficiente educación intercultural que aporte al desarrollo de destrezas. Esto nos conduce a la siguiente interrogante ¿cuáles son las conductas que debe poseer un perfil docente que responda a las necesidades educativas de un contexto rural, bilingüe e intercultural?

Por la problemática observada se propone responder a las necesidades educativas de los estudiantes en los subniveles de básica elemental y básica media, de la Escuela Ika, a través del análisis del entorno escolar de la comunidad Waorani de Guiyero y la construcción de un perfil docente intercultural bilingüe.

En cuanto al diseño de investigación, se trata de una propuesta teórico-conceptual sobre el perfil docente de un profesor intercultural bilingüe para la Escuela Ika. Para ello, se partió de la identificación de los hábitos de enseñanza de los profesores que actualmente laboran en esta institución y se contrastó con el desempeño de los educandos.

Es importante mencionar que no existe un estudio en el contexto de la comunidad Waorani con respecto al perfil docente intercultural bilingüe y se ve la necesidad de la elaboración del mismo, para guiar el quehacer del docente de la Escuela Ika. La ventaja que se obtendría de este estudio sería tener un perfil acorde a las necesidades del contexto cultural que pueda transferirse a otros espacios con características similares.

El perfil docente, según Gutiérrez y Langarica (citado por Dimas y Treviño, 2015), es un "instrumento estratégico de la planeación académica, diseñado para configurar las características relevantes de los elementos: profesor y alumno, en relación con los objetivos del proceso educativo" (p. 1666). Se pretende que el producto de esta investigación sirva de guía para la identificación de temáticas de capacitación para los actuales y futuros maestros involucrados en la educación intercultural bilingüe. Además, de que se involucre el aprendizaje y el manejo de estrategias innovadoras, propias de la sociedad del conocimiento y aptas para el desarrollo de destrezas, y se fomente el pensamiento lógico, crítico y creativo en los alumnos, junto con habilidades sociales y de trabajo autónomo y que respete y promueva la cultura y lengua de pueblos desfavorecidos y posiblemente en vías de desaparición.

La definición de las características importantes del perfil docente para la comunidad Waorani se espera no solo beneficie a la educación de los estudiantes de la Escuela Ika, sino que aporte al desarrollo de niños y jóvenes que se 
encuentran en situaciones de vulnerabilidad, mantienen un reducido contacto con las grandes ciudades y han sido desprovistos de una educación formal que les permita manejar habilidades para desempeñarse en un mundo laboral. Finalmente, se espera que el perfil docente sirva como referencia, para la creación de otros documentos similares y sea tomado en cuenta el momento de realizar nuevas contrataciones de profesores, para estas comunidades y otras con rasgos comunes.

La labor que realiza un docente en el aula está enmarcada por un perfil específico con elementos fundamentales donde interviene la relación estudiante-profesor para incidir en los procesos de enseñanza-aprendizaje. Este perfil está constituido por valores, actitudes, conductas y pautas de comportamiento como lo señala Jiménez (2007). El perfil docente debe estar contextualizado a los cambios o retos de la sociedad, ya que el mismo debe repercutir en su desarrollo científico y técnico. Es importante recordar que cada mensaje o actitud del profesor influye para en los estudiantes. Es por ello que el perfil de todo maestro debe construirse en proceso de cambio constante para responder a las necesidades de la época y de la sociedad.

En el contexto de la educación existe siempre la inquietud por cómo debe ser y actuar un docente, así como cuáles deberían ser sus competencias y funciones en el ámbito que se desenvuelve. El educador del siglo XXI debe motivar a los estudiantes a aprender, por ello requiere "... una concepción del proceso de aprendizaje que facilite la adquisición de las capacidades, proporcione las estrategias más generalizables para solucionar problemas y desarrolle capacidades socio-afectivas, tales como valores, actitudes, motivaciones y emociones" (Galvis, 2007, p. 49).

El perfil actual del docente requiere de nuevas estrategias, experiencias, valores, destrezas que le permitan enfrentar la realidad actual de la sociedad del conocimiento donde la tecnología cada día avanza y demanda nuevas competencias del estudiante y del maestro. Para esto, se necesita cambiar de paradigma educativo a uno donde el docente sea capaz de identificar las capacidades requeridas por un grupo social determinado, lo que permitirá pertinencia social a este nuevo perfil (Galvis, 2007).

Existen varios estudios donde se señala que para definir la profesión docente de la sociedad del siglo XXI hay que aceptar el desafío de ampliar el horizonte cultural e intervenir activa y comprometidamente como ciudadanos en el mundo actual. Para ello, Perrenoud (citado por Galvis, 2007) señala las competencias que debe poseer un docente bajo este perfil: "persona creíble, me- 
diador intercultural, animador de una comunidad educativa, garante de la ley, organizador de una vida democrática, conductor cultural e intelectual" (p. 50). Igualmente, Braslavsky (citado por Galvis, 2007) añade que los profesores para cumplir las funciones que el mundo actual demanda deben:

Planificar y conducir movilizando otros actores, adquirir o construir contenidos y conocimientos a través del estudio o la experiencia, identificar los obstáculos o problemas que se presentan en la ejecución de proyectos $u$ otras actividades del aula. Seleccionar diferentes estrategias para el desarrollo del proceso de enseñanza y aprendizaje, para la optimización del tiempo, de los recursos y de las informaciones disponibles (p. 51).

El proceso de construcción de un perfil docente basado en competencias para un contexto intercultural necesita de un cambio en la concepción del profesor. En este sentido, se puede alcanzar la transformación del maestro promoviendo las competencias de investigación que le permitan ayudar a los estudiantes a que se sitúe el currículo y la pedagogía en sus contextos socio históricos. Además, la formación integral del docente tiene que capacitarlo para desarrollar competencias sociales e interpersonales de los estudiantes a través de proyectos comunes que los preparen para resolver conflictos respetando los valores de cada uno.

Según la Declaración Universal de Derechos Humanos, ya en el año 1948, se determina que "toda persona tiene derecho a la educación" (UNESCO, 2011, p. 7), lo cual "favorecerá la comprensión, la tolerancia y la amistad entre todas las naciones y todos los grupos étnicos o religiosos" (UNESCO, 2016, p. 22) y promoverá el respeto por la identidad cultural, la lengua originaria y los valores axiológicos de cada pueblo (UNESCO, 2006). A su vez, la Declaración de las Naciones Unidas, sugiere que todos los niños y adolescentes reciban una educación, en todos los niveles, inclusiva, equitativa, de calidad y capaz de fortalecer la cultura e idioma de cada población y que no se permita cualquier tipo de discriminación (Naciones Unidas, 2007).

En cuanto a las demandas de la sociedad actual la UNESCO (s.f.) resalta que "la educación es esencial para un desarrollo humano, inclusivo y sostenible promovido por sociedades del conocimiento capaces de enfrentar los desafíos del futuro con estrategias innovadoras" (p. 48). Por otro lado, al referirse al contexto ecuatoriano, el Plan Nacional del Desarrollo 2017-2021 (Senplades, 2017) establece como política quinta del pri- 
mer objetivo: "Fortalecer el sistema de inclusión y equidad social, protección integral, protección especial, atención integral y el sistema de cuidados durante el ciclo de vida de las personas, con énfasis en los grupos de atención prioritaria, considerando los contextos territoriales y la diversidad sociocultural" (p. 58) y como objetivo segundo: "Afirmar la interculturalidad y plurinacionalidad, revalorizando las identidades diversas" (p. 60) y entre sus políticas: "Garantizar la preservación de las lenguas tradicionales, el multilingüismo y el sostenimiento de sistemas de educación intercultural y conocimiento de las diversidades ancestrales" (p. 63).

Asimismo, la Constitución de la República del Ecuador (2008), en el artículo 26, señala que "la educación es un derecho de las personas a lo largo de su vida y un deber ineludible e inexcusable del Estado" (Constitución de la República del Ecuador, 2008, p. 16). Y la Ley Orgánica de Educación Intercultural (LOEI), en el artículo 2, literal w:

Garantiza el derecho de las personas a una educación de calidad y calidez, pertinente, adecuada, contextualizada, actualizada y articulada en todo el proceso educativo, en sus sistemas, niveles, subniveles o modalidades; y que incluya evaluaciones permanentes. Así mismo, garantiza la concepción del educando como el centro del proceso educativo, con una flexibilidad y propiedad de contenidos, procesos y metodologías que se adapte a sus necesidades y realidades fundamentales. Promueve condiciones adecuadas de respeto, tolerancia y afecto, que generen un clima escolar propicio en el proceso de aprendizaje (LOEI, 2011, p. 10).

Bajo las políticas y propósitos enunciados, el Ministerio de Educación del Ecuador (MINEDUC), en el año 2013, publica el Modelo de Educación Intercultural Bilingüe (MOSEIB) y en 2016, presenta el Currículo Nacional de EGB y BGU (2016a), documento obligatorio para la educación formal en todo el país. Más tarde, la Subsecretaría de Educación Intercultural Bilingüe, propone el Currículo Intercultural Bilingüe, el cual promueve la interculturalidad y fue traducido a las catorce lenguas aborígenes del Ecuador, entre ellas el waotededo. 


\section{MATERIALES Y MÉTODOS}

Fue necesario aplicar instrumentos aptos para identificar las destrezas alcanzadas, en las áreas de Matemática y Lenguaje, por los niños de la Escuela Ika y listas de cotejo para la sistematización de la observación de hábitos de trabajo de los alumnos, datos que permitieron detectar la situación actual de la educación en las comunidades waorani. Por otro lado, con la finalidad de visualizar la realidad de los docentes de la Escuela Ika se emplearon fichas de observación y entrevistas a docentes, instrumentos que midieron los hábitos de trabajo de los profesores en el aula, sus habilidades y estrategias metodológicas aplicadas. Asimismo, se analizó el Currículo Nacional de EGB y BGU, el perfil de salida de los estudiantes de Bachillerato y la construcción y validación de perfiles profesionales educativos. Todo ello para la elaboración de un documento que integre las competencias específicas que debe poseer el docente intercultural bilingüe.

Respecto a la población de la investigación, está compuesta por 47 estudiantes que pertenecen a las comunidades de Timpoka, Ganketepare, Peneno, Paraíso y Guiyero, de los cuales 20 formarán parte de la muestra, correspondiente a estudiantes de los niveles de 3ro, 4to, 5 to y 6 to de EGB. En cuanto a los docentes, todos ellos constituirán la muestra, tres bachilleres y una persona con título de cuarto nivel.

Para la determinación del perfil docente se emplearán tres técnicas de medición:

a. "Observación naturalista": diseñada para obtener información relacionada con una estructura académica administrativa, infraestructura y recursos didácticos, aspectos físicos del aula, aspectos curriculares y aspectos socioeconómicos sin afectar la dinámica cotidiana de la institución.

b. "Observación estructurada": mediante el uso de instrumentos de listas de cotejo para la observación de clase y registros de hábitos del trabajo que identifican las dinámicas escolares, así como las estrategias de enseñanza y evaluación y los recursos didácticos empleados por los docentes de los niveles de EGB de la Escuela IKA.

c. "Entrevistas semiestructuradas": a través de preguntas predeterminadas aplicadas a la población de maestros de la EGB de la Escuela IKA. 


\section{RESULTADOS}

Por medio de la aplicación de los instrumentos detallados en párrafos anteriores se identificó las características de la escuela waorani. A continuación, se describe el contexto:

El desarrollo de iniciativas educativas en las comunidades waorani, ubicadas en el interior del Parque Nacional Yasuní, se han desarrollado gracias al aporte de organizaciones de misioneros, empresas petroleras, organizaciones no gubernamentales, organizaciones comunitarias y universidades que, sin ser el órgano rector de la educación intercultural bilingüe en el Ecuador, han aportado con su colaboración para el pueblo waorani. Por tal motivo, existe la posibilidad de gestión de recursos económicos que permitan trabajar con esta nacionalidad, que provienen de los actores directos e indirectos localizados en el Parque Nacional Yasuní y el correspondiente acompañamiento e intervención del Ministerio de Educación como órgano rector de la educación en el Ecuador.

En cuanto al acceso a la región, el Parque Nacional Yasuní es el área protegida más grande del Ecuador continental ubicado en las provincias de Pastaza y Orellana, entre el río Napo y el río Curaray designado por la UNESCO, como una Reserva de la Biosfera y parte del territorio donde se encuentra ubicada la nacionalidad waorani. El Centro Educativo Comunitario Intercultural Bilingüe Ika de la comunidad waorani de Guiyero, objeto de este estudio, está localizado en el interior del PNY y su acceso se realiza a través del cruce del río Napo, en la localidad de Pompeya de la parroquia Alejandro Labaka a 32 km de la vía Maxús hacia el sureste. La mencionada vía es la ruta de ingreso a los campos de explotación petrolera de la Compañía REPSOL.

En el Parque Nacional Yasuní se encuentran localizadas algunas comunidades de la nacionalidad Waorani, una minoría étnica ancestral en el contexto de la Amazonía ecuatoriana. Históricamente han quedado relegadas a la periferia de los intereses de la sociedad nacional, desatendidas desde las primeras etapas de desarrollo, en las que fueron contactados y desde que se inició el denominado proceso de "pacificación" en 1958. Es notable que el tema educativo formal y no formal ha recaído, por lo general, en organizaciones de misioneros, empresas petroleras, organizaciones no gubernamentales, organizaciones comunitarias de base y otros actores, los cuales en la medida de sus posibilidades, han tratado de suplir la ausencia del estado con su relativa presencia. No obstante, las demandas de la educación en este sector del país siguen siendo grandes. 
La Escuela lka es atendida por dos maestros asignados oficialmente por el Ministerio de Educación y un docente determinado por la compañía ENTRIX, que brinda servicios a la empresa petrolera Repsol. En cuanto al nivel de escolaridad, de acuerdo con los datos poblacionales de Guiyero, Timpoka, Paraíso y Ganketapare, 47 habitantes forman parte del sistema de educación y están inscritos en alguno de los niveles de educación. Al realizar una serie de pruebas básicas en distintas asignaturas, matemática, lenguaje, ciencias naturales y sociales, se pudo constatar que los alumnos de los diferentes niveles tuvieron un rendimiento regular o insuficiente. Por otra parte, el 7,19\% de la población culminó solo la primaria, el 7,19\% la secundaria y sólo el 0,72\% culminó la universidad (una persona, la que se desempeñaba como Director del establecimiento educativo en el año 2016). Un 7,91\% desertó de la Educación General Básica y un 17,27\% abandonó el bachillerato. Un 5,04\% de la población en edad escolar no asiste a la escuela o colegio, un 9,35\% son analfabetos y un 11,51\% son infantes que aún no alcanzan la edad escolar.

En el área es posible evidenciar: (a) un déficit de recursos humanos para impulsar una educación bilingüe-intercultural con calidad, puesto que el cuerpo docente tiene una escasa formación y no recibe capacitación continua; (b) una gestión inadecuada y discontinua del currículo, dada por la falta de contextualización del Currículo Nacional y los materiales educativos a las condiciones socio-culturales, lingüísticas y ambientales de la población y a las necesidades individuales de los alumnos; (c) irregularidad en el proceso de enseñanza-aprendizaje, debido a la falta de una cultura de planificación en el cuerpo docente, el cual no define una programación anual y diaria conduciendo a la pérdida de tiempo efectivo para la enseñanza, problema que además se agrava por el repetido ausentismo del cuerpo docente y discente, ocasionado por la falta de supervisión y acuerdos orientados a balancear el calendario escolar con las dinámicas sociales y culturales (fiestas) y de la economía comunitaria (caza, pesca, recolección); (d) inadecuados y/o insuficientes recursos físicos y tecnológicos en el establecimiento educativo en el que funciona la escuela y colegio; y (e) proceso educativo desvinculado de la comunidad.

Por consiguiente, el nivel de la calidad de la educación es deficiente y alejado de la realidad sociocultural y ambiental de las comunidades Waorani. Las principales consecuencias del problema se manifiestan en los bajos niveles de escolaridad y rendimiento académico que se han observado; la desmotivación e inhibición del aprendizaje, que a su 
vez ocasiona rezago educativo, fracaso y deserción escolar; la inhibición del desarrollo cognitivo, emocional, social de los estudiantes; el estancamiento en la reducción del analfabetismo; el fomento de la pérdida de la identidad y de los saberes ancestrales, lo cual a su vez conduce a la aculturación; el riesgo de perder el waotededo como lengua primaria ancestral; el escaso aporte a la generación de conciencia ambiental y a la valoración del patrimonio natural, lo que a su vez propicia la sobreexplotación de los recursos de la vida silvestre y la pérdida de la biodiversidad; la dependencia de modelos asistencialistas; la adopción de hábitos adversos como el alcoholismo, violencia e irresponsabilidad; y la mínima inserción laboral. Estos efectos merman la calidad de vida de la población y agravan las condiciones de inequidad y la exclusión social de este grupo étnico minoritario.

Los estudios referentes a la comunidad Waorani son muy reducidos. No obstante, es importante resaltar la investigación realizada por Laura Rival (1996) quien menciona algunos detalles de las formas de aprendizaje de un grupo waorani, en ese momento, recién contactados por la civilización. Sin embargo, se debe indicar que su trabajo muestra factores ya no presentes en los pobladores de Guiyero, quienes han cambiado sus hábitos de vida en estos treinta años. También pueden aportar los estudios de Flores (2016), a través de la Atención educativa a la nacionalidad Waorani: pautas para mejorar la calidad educativa.

\section{DISCUSIÓN}

Para la construcción del perfil docente intercultural bilingüe se consideró el contexto educativo de la Escuela Ika y los componentes detallados por el Ministerio de Educación para la construcción y validación de perfiles profesionales educativos. En este documento se sugiere la elaboración de varios perfiles profesionales, entre ellos, el Educación Generalista de EGB de 2do a $7 \mathrm{mo}$ grado y otros orientados a la educación inclusiva, no obstante, se ha dejado de lado la educación intercultural bilingüe.

La construcción y validación de perfiles profesionales educativos considera los lineamientos establecidos en el Plan Nacional de Desarrollo (2013-2017), la Ley Orgánica de Educación Intercultural (LOEI) y la Ley Orgánica de Educación Superior donde se describen las políticas 
públicas del estado ecuatoriano en relación a la educación y al rol que debe cumplir el docente.

Para el desarrollo del perfil docente intercultural bilingüe se incluyó algunas de las directrices del Plan Nacio- nal de Desarrollo, la LOEI, los Estándares de Desempeño Docente y el Modelo de Educación Intercultural Bilingüe (MOSEIB) y los requerimientos y necesidades de los docentes dentro del contexto específico de la Escuela Ika.

\section{PROPUESTA DEL PERFIL DOCENTE INTERCULTURAL BILINGÜE}

Para la elaboración del perfil intercultural bilingüe se clasificó las características del maestro dentro de cada uno de los siguientes componentes: identidad profesional, desarrollo profesional, gestión de enseñanza- aprendizaje y dis- ciplinar propuestos por el Ministerio de Educación. (MINIDUC, 2016b).

A continuación, se enuncian las características para el profesor de educación intercultural bilingüe:

\section{IDENTIDAD PROFESIONAL}

Este componente permite la autovaloración que el maestro puede percibir de su persona, ante el desarrollo integral de sus educandos.

- Actúa con compromiso ético ante su propio desempeño personal.

- Responde con compromiso ético ante el desarrollo integral de sus estudiantes favoreciendo la formación de líderes.
Lidera el proceso educativo en función de solución de problemas en la comunidad.

- Promueve el cuidado del ambiente natural a través de su propio ejemplo. Mantiene una comunicación asertiva con los padres de familia y líderes de la comunidad.

- Emprende esfuerzos para mejorar la calidad de educación del entorno en que se desenvuelve. 
- Mantiene confidencialidad con la documentación de la Institución.

- Respeta la cultura del grupo humano con el que realiza su labor do- cente a través de la promoción de la democracia, derechos y responsabilidades.

\section{DESARROLLO PROFESIONAL}

Este componente considera las tareas que el docente realiza para mejorar, actualizar y perfeccionar su saber hacer en el aula.

- Muestra interés por capacitarse en las áreas del conocimiento que imparte.

- Participa de cursos de actualización de estrategias pedagógicas.

- Investiga aspectos relacionados con la cultura del entorno que imparte las clases.
- Conoce y aplica el idioma nativo del contexto donde se desenvuelve como docente.

- Actualiza sus aprendizajes sobre ajustes curriculares para la inclusión.

- Perfecciona sus técnicas para el manejo de la escuela rural: unidocente o pluridocente.

- Mejora sus herramientas para la gestión educativa.

- Renueva sus conocimientos a través de cursos de aplicación de Tic en el aula.

\section{GESTIÓN DE ENSEÑANZA APRENDIZAJE}

Este componente aborda el desenvolvimiento del docente en el proceso de enseñanza aprendizaje a través de tres fases: preparación, ejecución y evaluación.

- Planifica las clases en función de las necesidades, intereses y contexto cultural de los estudiantes bajo los formatos establecidos por las entidades competentes.
- Elabora material didáctico acorde a la temática de la clase.

- Aplica estrategias metodológicas acordes al desarrollo psicológico, cognitivo y afectivo de los estudiantes que promuevan el pensamiento lógico, crítico y creativo.

- Domina conocimientos propios de las áreas que imparte.

- Ejecuta ajustes curriculares en rela- 
ción de las necesidades de los niños y el entorno cultural.

- Emplea Tecnologías de la Comunicación como apoyo a su gestión en el aula.

- Aplica estrategias para el manejo de grupos de distintos grados en el mismo salón.

- Utiliza un lenguaje claro y sencillo para la comprensión de los contenidos pedagógicos.

- Integra actividades que fomenten las habilidades sociales y el trabajo autónomo.
- Explica los criterios de evaluación que empleará durante el proceso educativo.

- Recoge conocimientos previos para la contextualización del aprendizaje.

- Promueve la expresión de ideas propias respetando los puntos de vista de todos.

- Aprovecha elementos del entorno para propiciar un aprendizaje significativo.

- Emplea actividades de evaluación acordes a las destrezas trabajadas durante el proceso didáctico.

\section{DISCIPLINAR}

Este componente destaca cumplimiento de normas y reglamentos establecidos en el Ministerio de Educación e institución donde el docente labora.

- Cumple con la entrega de planes meso y micro curriculares en los plazos establecidos por la institución y bajo los formatos establecidos por el Currículo Nacional de Educación Intercultural Bilingüe.

- Reporta las calificaciones del grupo a cargo en los tiempos previstos por las autoridades institucionales.
- Lidera reuniones con padres de familia y la comunidad para resolver problemas escolares.

- Informa de manera oportuna las situaciones de estudiantes con dificultades en el aprendizaje y/o en la conducta.

- Envía la documentación que solicita el Ministerio de Educación para el seguimiento y promoción de los estudiantes.

- Aplica el código de convivencia elaborado por el centro educativo donde trabaja. 


\section{CONCLUSIONES}

Políticas educativas nacionales e internacionales, en los últimos años, enfatizan la importancia de incluir en sus programas educativos directrices orientadas al respeto y valoración de la cultura y las lenguas originarias. Entidades como las Naciones Unidas y el Ministerio de Educación del Ecuador mediante su normativa promueven el respeto a los derechos de los pueblos indígenas y para ello han elaborado recursos pedagógicos contextualizados a las necesidades de estos grupos.

La comunidad waorani es un ejemplo de un pueblo indígena que requiere de una educación con carácter intercultural y bilingüe asociada a la lengua waotededo. Actualmente, los docentes de este contexto provienen de otras regiones del país, por lo que desconocen la lengua y la cultura. Además cuentan con una escasa preparación académica que les permita desenvolverse con estrategias pedagógicas acordes a las necesidades de la población. Por tal motivo, urge establecer lineamientos que permitan identificar el rol del docente en este ámbito educativo.
Con el fin de establecer las conductas que deberá poseer el maestro intercultural bilingüe se vio la necesidad de elaborar un perfil docente acorde al entorno y enmarcado en las directrices establecidas por la Constitución del Ecuador, la LOEI, el Currículo Nacional, MOSEIB y el documento para la construcción y validación de perfiles profesionales educativos.

La propuesta incluye los componentes de: identidad profesional, desarrollo profesional, gestión de enseñanza aprendizaje y disciplinar y en cada uno de ellos se establecen las conductas que un maestro de educación intercultural bilingüe deberá considerar para su labor docente dentro del aula, el centro educativo y la comunidad a la que ha sido asignado.

Este artículo informa sobre las dinámicas actuales que se llevan en la Escuela lka y pretende originar cambios en el rol que cumple el docente intercultural bilingüe con la finalidad de dar respuesta a las necesidades particulares de este sector alejado de las grandes ciudades y que espera mayor atención por parte de las autoridades nacionales. 


\section{REFERENCIAS}

Alfaro Rodríguez, Magdalena; Gamboa Jiménez, Alejandra; Jiménez Sánchez, Susana; Pérez, Jorge Martín; Ramírez González, Andrea; Vargas Dengo, Marie-Claire. ( 2008). Construcción del perfil profesional docente de sétimo año: respuesta a una necesidad actual Revista Electrónica Educare, 12 (2) ,pp. 31-45 Recuperado de: http://www.redalyc.org/ pdf/1941/194114586005.pdf

Constitución de la República del Ecuador. (2008). Constitución de la República del Ecuador. Recuperado de: https://www.oas.org/juridico/ pdfs/mesicic4_ecu_const.pdf

Dimas, M. y Treviño, A. (2015). La importancia de la definición del perfil docente de una institución de educación superior en Ingeniería. Vinculatégica, 1(1), 1662-1680. Recuperado de http://www. web.facpya.uanl.mx/vinculategica/Revistas/1662-1680\%20 LA\%2OIMPORTANCIA\%20DE\%20 LA\%20DEFINICION\%20DEL\%20 PERFIL\%20DOCENTE\%20DE\%20 UNA\%2OINSTITUCION\%20DE\%20 EDUCACION\%2OSUPERIOR\%20 EN\%2OINGENIERIA..pdf

Flores, J. A. (2016). Atención educativa a la nacionalidad Waorani: pautas para mejorar la calidad educativa. Buenos Aires, Argentina: CLACSO Galvis, R. V. (2007). De un perfil docente tradicional a un perfil docente basado en competencias. Acción Pedagógica, (16), 48 - 57.

MINEDUC. (2013). MOSEIB: Modelo de Educación Intercultural Bilingüe. Recuperado de https:// educacion.gob.ec/wp-content/ uploads/downloads/2014/03/ MOSEIB.pdf

MINEDUC. (2016 a). Currículo Nacional de EGB y BGU. Recuperado de https://educacion.gob.ec/ wp-content/uploads/downloads/2016/03/Curriculo1.pdf

MINEDUC. (2016 b). Construcción y validación de perfiles profesionales educativos. Recuperado de www. ces.gob.ec/doc/cuartoseminario/ perfiles\%20ministerio\%20de\%20 educacion.pdf

MINEDUC. (2017). Currículo Nacional Intercultural Bilingüe: Neete mea tededo tedeyomo. Recuperado de https://educacion.gob.ec/ wp-content/uploads/downloads/2017/03/WAO_CNIB_2017. pdf

Naciones Unidas. (2007). Declaración de las Naciones Unidas sobre los derechos de los pueblos indíge- 
nas. Recuperado de http://www. un.org/esa/socdev/unpfii/documents/DRIPS_es.pdf

Naciones Unidas. (2016). Agenda 2030 y los Objetivos de Desarrollo Sostenible: Una oportunidad para América Latina y el Caribe. Recuperado de http://www. sela.org/media/2262361/agenda-2030-y-los-objetivos-de-desarrollo-sostenible.pdf

Pontificia Universidad Católica del Ecuador. (2016). Waorani Minkayonta. Recuperado de https://minkayonta.wordpress.com/fortalecimiento-del-talento-humano/

LOEl. (2011). Ley Orgánica de Educación Intercultural. Recuperado de https://oig.cepal.org/sites/default/ files/2011_leyeducacionintercultural_ecu.pdf

Rival. L. (1996). Hijos del sol, padres del jaguar: los huaorani de ayer y hoy. Quito, Ecuador: Abya-Yala.

Secretaría Nacional de Planificación y Desarrollo. (2017). Plan Nacional del Desarrollo 2017-2021. Recuperado de http://www.planificacion. gob.ec/wp-content/uploads/
downloads/2017/10/PNBV-26OCT-FINAL_OK.compressed 1.pdf UNESCO. (2006). Directrices de la UNESCO sobre la educación intercultural. Recuperado de http://unesdoc.unesco.org/images/0014/001478/147878s.pdf

UNESCO. (2011). La UNESCO y la Educación: "Toda persona tiene derecho a la Educación".Recuperado de http://unesdoc.unesco.org/ images/0021/002127/212715s. pdf

UNESCO. (2016). Estrategias didácticas: guía para docentes de educación indígena. Recuperado de http:// www.unesco.org/fileadmin/MULTIMEDIA/FIELD/Mexico/images/ Publicaciones/GUIADOCENTE2016.pdf

UNESCO.(s.f). Indicadores Unesco de cultura para el desarrollo. Recuperado file:///C:/Users/ MARROYO621/Desktop/Tutor\%C3\%ADas/201803(\%20semipresencial\%20y\%20presencial)/ Educacion\%20(1).pdf 